\title{
Experimental Study on Active Control of Surge in a Centrifugal Compression System*
}

\author{
NIE CHAOQUN ${ }^{\dagger}$, CHEN JINGYI and CHEN NAIXING \\ Institute of Engineering Thermophysics, Chinese Academy of Sciences, P.O. Box 2706, Beijing 100080, P.R. China
}

(Received 24 April 1998; In final form 10 March 1999)

\begin{abstract}
An experimental study has been carried out on the active control of surge in a centrifugal compression system. With a computerized on-line control scheme, the surge phenomenon is suppressed and the stable operating range of the system is extended. In order to design the active control scheme and choose the desired parameters of the control system inputs, special emphases have been placed on the development of surge inception and the nonlinear interaction between the system and the actuator. By use of the method designed in the present work, the results of active control on surge have been demonstrated for the different $B$ parameters, different prescribed criteria and different control frequencies.
\end{abstract}

Keywords: Compressor, Aerodynamics, Experimental study, Surge, Flow instability, Active contro

\section{INTRODUCTION}

Similar to the situation of aeronautical propulsion, it is well known that the surge phenomenon may seriously hinder industrial fans from operating steadily and improving in behavior. In order to ensure the safe operation of compression systems, for a long time industrial fans are kept away from operating under high loading. Therefore, the possibility of high-pressure ratio or high efficiency is sacrificed.

Since Epstein et al. (1989) first put forward a preliminary active control plan to suppress instability in turbomachinery, it appears possible that active control can be used to maintain the operation of a compression system at high parameters without having to change the original set-up of the compression system. Subsequently, Ffowcs Williams and Huang (1989) obtained the results of active control of surge in the centrifugal compressor by adopting the principle of anti-sound in acoustics. At the same time, Gysling et al. (1991) made use of the aeromechanical feedback principle of the special spring-mass-damper's perturbation response to the compression systems, and Pinsley et al. (1991) took advantage of the method of close-coupled throttle obtaining more satisfied control results. On the

\footnotetext{
* This paper was originally presented at ISROMAC-7.

${ }^{\dagger}$ Corresponding author. Tel.: +86-10-62560740. Fax: +86-10-62575913.
} 
other hand, in recent years the successful experiments on surge also stimulate the rapid development of the active control and understanding about the inception mechanism of rotating stall (Garnier et al., 1991; Day, 1993a; Hoying, 1993; Tryfonidis et al., 1995). A number of results on active stabilization of rotating stall have been obtained. It is obvious that the theoretical and experimental analyses on active control of flow instabilities are important areas of investigation in turbomachinery.

In design of active control programs, there are two distinct methods. The first method consists of transducers, DAQ devices, a computer and actuators. The main feature of this method is electromechanical feedback (Pinsley et al., 1991; Day, 1993b; Paduano et al., 1993; Haynes et al., 1994). In the second method, the principle of aeromechanical feedback is applied to reach the objective of the active control to flow instabilities. The control method is strongly related with unsteady dynamic responses in the system (Gysling et al., 1991; Gysling and Greitzer, 1995). It seems that the first method is practical for surge while the second is suitable for rotating stall.

The objective of this project is to suppress surge with the technique of active control, in order to expand the steady-state safe operating range. Also, the nature of the inception process of surge and the nonlinear interaction between the system and the actuator are investigated in detail.

\section{EXPERIMENTAL FACILITIES AND CONTROL SYSTEMS}

\section{Experimental Rig}

As the experimental research is closely related to the actual engineering application, the special centrifugal fan is chosen to be a testing rig. The surge phenomenon easily occurs near the surge boundary line. According to the Moore-Greitzer model (Moore and Greitzer, 1986a,b), and the criterion for the $B$ parameter (Greitzer, 1976a,b; 1981), the dynamic behavior of the fan can be simulated for the selected scales and shapes of the rig accordingly. The simulated results show that when $B$ parameter is greater than 0.25 , the surge occurs in the centrifugal compression system (Fig. 1). For acquiring control results at the different $B$ parameters, three cases for $B$ parameters $(B=0.25, B=0.28, B=$ $0.34)$ are designed. The experimental rig is composed of centrifugal fan, inlet duct, plenum and throttle (Fig. 2). The operating point of the system can be compelled into surge by decreasing the cross section of the throttle.

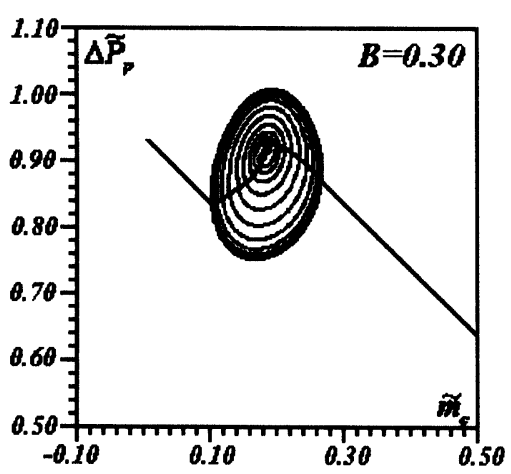

FIGURE 1 Simulated results. (The surge point of the system is simulated for designing the rig.)

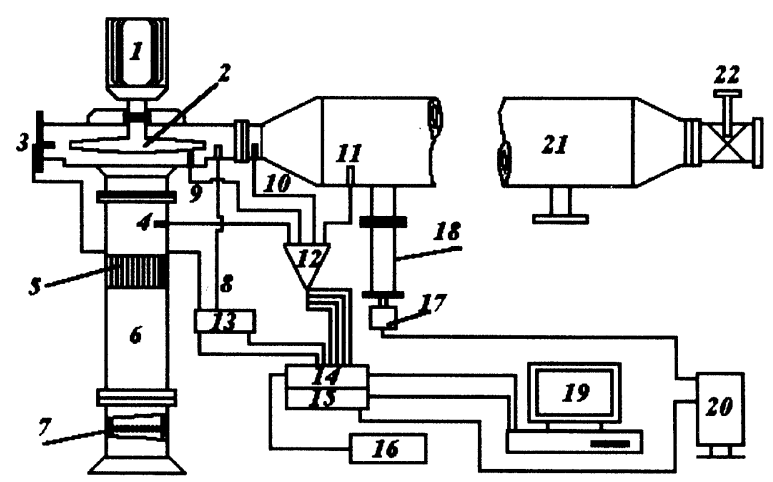

FIGURE 2 The structural outline of active control. (The experimental facility includes the compression system, the measuring and controlling system, and actuator.) 1. Motor, 2. Centrifugal Fan, 3. Hot Wire No. 1, 4. Sensor No. 1, 5. Rectifier Grids, 6. Inlet of Fan, 7. Flow Adjustable Nets, 8. Hot Wire No. 2, 9. Sensor No. 2, 10. Sensor No. 3, 11. Sensor No. 4, 12. Amplifier, 13. Anemometer, 14. A/D Board, 15. D/A Board, 16. Oscilloscope, 17. Servo Motor 18. Actuator, 19. Computer, 20. Servo Power, 21. Plenum, 22. Throttle. 


\section{Measurement Systems}

Four pressure transducers, a constant temperature anemometer, the high-performance data acquisition board, and the computer are set up for a realtime measuring and supervising system. In order to distinguish the behavior of dynamic response in inlet, plenum, radial and circumferential position of the centrifugal fan, the dynamic accuracies of the four transducers are checked carefully by using laser-tube instruments. It is found that the damping coefficient is equal to 0.0786 , the oscillating frequency is equal to $2150 \mathrm{~Hz}$, and the angle of lag phase retardation is equal to 0.015 . It is shown that the four transducers agree with the demands of supervision to the dynamic signal. Because of fragileness of the hot wire, it will not be used as the probe providing an input signal while exerting the plan of the active control.

\section{Control System}

The instruments and equipment in the actual control scheme include actuator, servo motor, actuating power supply, D/A board, the dynamic pressure transducers, the amplifiers and control software (Fig. 2). The electromechanical feedback system is employed to obtain a series of different control outcomes and to investigate the nonlinear properties between the system and the actuator.

\section{DYNAMIC BEHAVIOR AND INCEPTION DEVELOPMENT OF SURGE}

\section{Dynamic Behavior}

In order to suppress surge, the dynamic characteristics are investigated for different $B$ parameters. Figure 3 represents the compulsive surge process, and Figure 4 shows the spontaneous surge process. It is shown that the developmental time of the former is shorter than that of the latter from flow stability to flow instability, and the strength of the oscillation of the former process is stronger than that of the latter from the inception to full developed surge. The

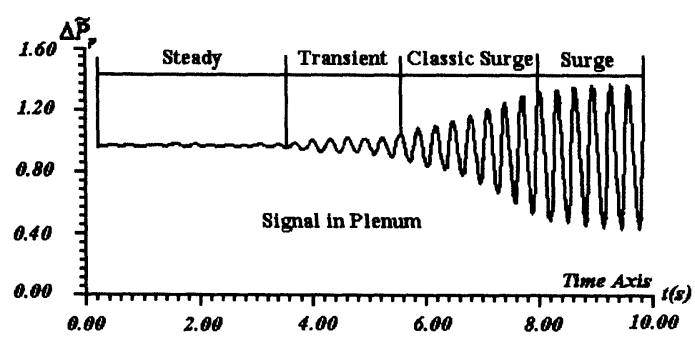

FIGURE 3 Compulsive surge $(B=0.28)$. (The transient process of surge with closing throttle continuously.)

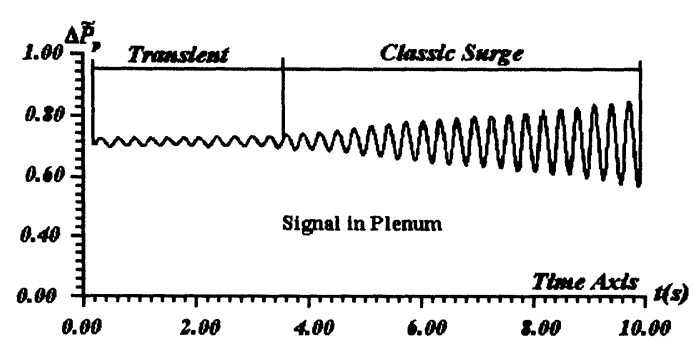

FIGURE 4 Spontaneous surge $(B=0.28)$. (The transient process of surge with closing throttle intermittently.)

strength of surge in the compression system increases while $B$ parameters become large (Fig. 5).

\section{Inception Process of Surge}

To implement the plan of active control on surge, the fundamental feature of the surge inception is investigated qualitatively. As soon as the small fluid perturbations, which are related to surge, occur, the amplitude of the perturbation will increase gradually, the stronger fluid oscillation will be incited rapidly, and surge will occur finally. Therefore, the question is whether it is possible to put the control measure into effect well before this classical surge appears in the system. The better on-line method is based on diagnosing the inception. Figure 6 shows the frequency spectrum of the inception development in the plenum for the compulsive surge. The whole time-resolved dynamic process is divided into four pieces (1, 2, 3 and 4). Each piece includes 1024 discrete points. Then successive frequency analysis is used to examine the behavior of the four pressure data sets individually. From the development of the 


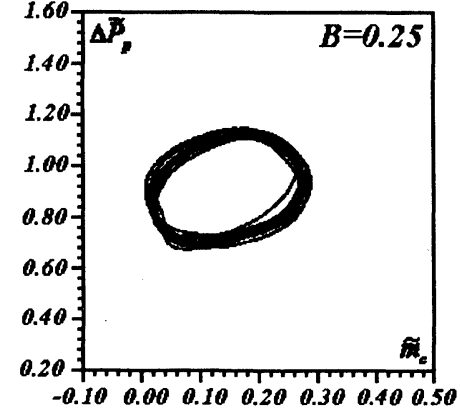

a
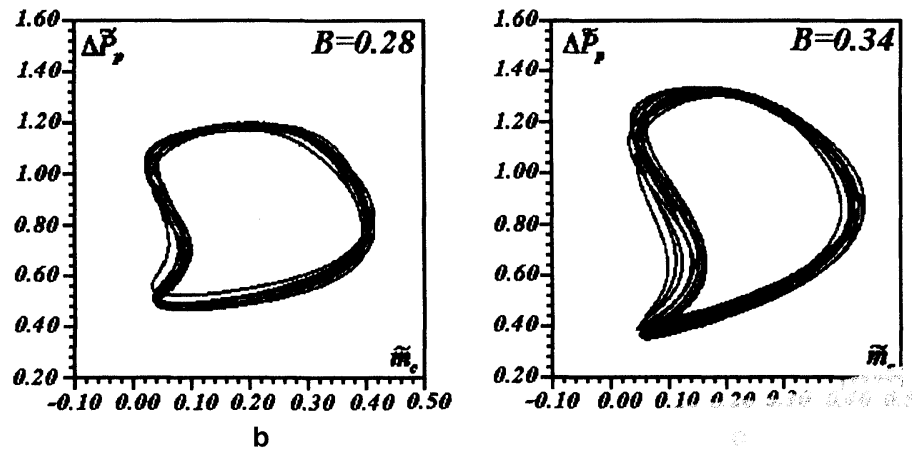

b

FIGURE 5 Surge limited cycles at three different $B$ parameters. (The dynamic characteristic of su. facility, the strength of surge oscillation increases while $B$ parameters become larger.)

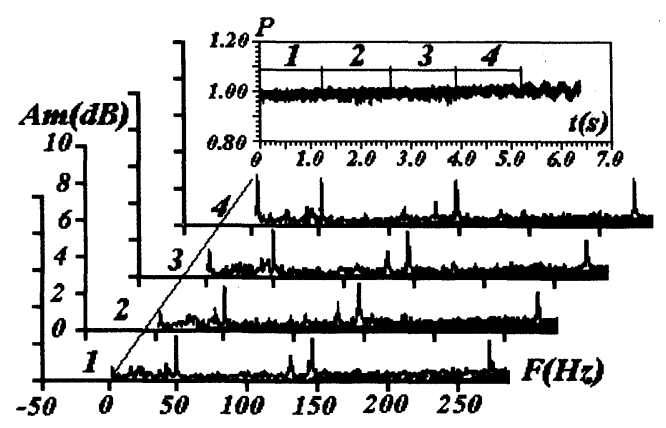

FIGURE 6 Surge inception. (The identification of surge inception is based on the divided frequency spectrum.)

low frequency signal in Fig. 6 , the $2.71 \mathrm{~Hz}$ signal increases successively and is detectable at data set number 2, about $4 \mathrm{~s}$ before surge. Therefore, this low frequency signal could be considered as a warning before surge. The measurement of the inception process based on the divided frequency spectrum analysis consists of the first step to design the active control plan and transitional time from the flow stability to the flow instability provides the enough time needed for the data processing and analyzing.

In addition, the characteristics of the surge inception development are also investigated under the three different $B$ parameters and the different positions of pressure measurement in detail. It is shown that the surge inception signal is weak at both the inlet and the circumference of the centrifugal fan, but it is strong and clear at both the outlet of the centrifugal fan and the plenum during the same moment. When the control scheme is implemented, the signal at either the plenum or the outlet of the centrifugal fan is chosen to be the input signal for the closed control loop.

\section{NONLINEAR INTERACTION WITHIN ACTUATOR AND SYSTEM}

Many different forms of actuators are proposed by Simon et al. (1993). In recent years, the effect of stabilization on flow instability has been achieved with the actuators (Ffowcs Williams and Huang, 1989; Gysling et al., 1991; Day, 1993b; Paduano et al., 1993; Gysling and Greitzer, 1995). The used actuators have one common feature. They can produce the small controlled friendly disturbances created by the control model. The disturbances provide damping action with amplitude development of surge and rotating stall.

In our experiment, the self-designed actuator of rotary valve type is driven by a servo motor with its rotational speed manipulated by the computer in the control loop. The actuator is to create the small friendly disturbances in the compression system. Figure 7 expresses the time-resolved dynamic signal of the pressure at the outlet of the centrifugal fan when the system is still away from the surge. These small disturbances are created by the actuator under the different speeds of the servo motor and supposed to suppress the initial perturbation of the surge. It is obvious from Fig. 7 that the wave 
from of the disturbances is not the ideal first harmonic wave form but including many complicated harmonics. Therefore, in order to select the needed controller's frequency, the nonlinear interaction between the actuator and the system are investigated. Here, the tendency of the first three harmonics is analyzed. The frequency as well as the amplitude characteristics of the nonlinear influence are shown in the Fig. 8 where the lower index "a" refers to the disturbance in the system created by the

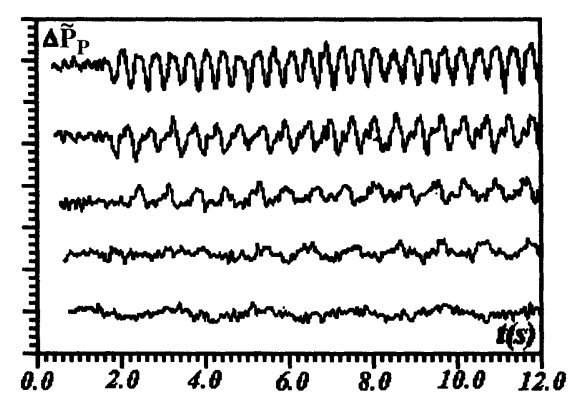

FIGURE 7 Small friendly disturbances. (The actuator provides the system with friendly disturbances at operating ranges.) actuator and "c" refers to the controlling frequency of the servo motor. From Fig. 8, both the frequency and amplitude values of the first harmonic wave of the disturbances increase sequentially with parabolic form when the controller's frequency is increased. The amplitude values of the first harmonic wave is close to be identical to each other when the controller's frequency is less than $50 \mathrm{~Hz}$ and the minimum value occurs when the controller's frequency is equal to about $20 \mathrm{~Hz}$. For the second harmonic wave, the variation tendency is nearly in agreement with that of the first harmonic. But when the controller's frequency is greater than $70 \mathrm{~Hz}$, the amplitude value of the second harmonic changes to a decreasing tendency. For the third harmonic, the changing tendency is similar to the harmonic sine wave and the wave disappears in the compression system when the controller's frequency is greater than $50 \mathrm{~Hz}$.

On the whole, these nonlinear characteristics between the actuator and the compression system must be taken into account in order to design the actual plan of the active control on surge. In our
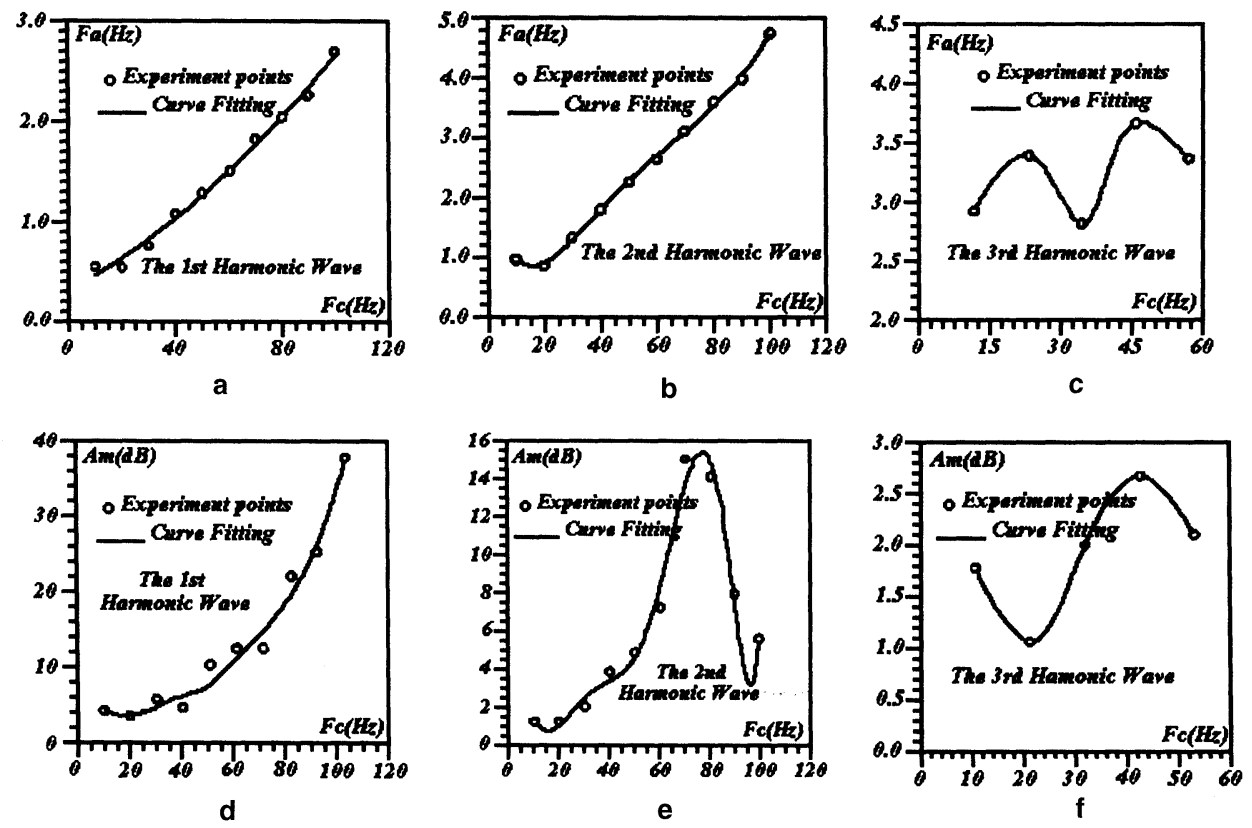

FIGURE 8 The characteristics of frequency and amplitude between the actuator and the system. (The controlled transformation of small friendly disturbances provided by actuator induces the nonlinear alternation of the system in frequency domain.) 
experiment, only the characteristic of the first harmonic wave is utilized to suppress the surge, but the nonlinear behaviors of the second and the third harmonics are also useful in understanding the nonlinear effect of the active control.

\section{EFFECTS OF ACTIVE CONTROL}

\section{The Designed Approach}

The objective to suppress the surge phenomenon is to restrict the amplitude of the fluid oscillation. In order to realize this objective, the effects of the small friendly disturbances are to weaken the amplitude of pressure and flow quantity oscillation from the inception. As a result, the operating ranges of the system can be expanded by exerting the small friendly disturbances from the actuator.

According to the analyzed results of the inception, the real-time signals from both the plenum and the outlet of the fan are chosen to be the input signal of the closed-loop control plan. At the same time, the adjusted pulse signal provided by the computer is elected to be the output. Within the technique of dynamic identification, the control model can be identified. Figure 2 stands for the structural outline of active control to surge. By decreasing the flow cross section in the throttle sequentially, the dynamic behavior of the system begins to work as an unsteady boundary. Then, acquiring the dynamic pressure signal continuously, obtaining the discrete variable data through the data amplifiers and A/D board, and conducting the real-time analyses and processing those data by computer. If the inception is detected, the computer immediately sends out a functional pulse signal, to rotate the servo motor at a specific function, finally to cause the actuator operating. The objective was achieved successfully.

\section{The Control Effects at the Different Control Frequencies}

The control frequency is governed by the pulse frequency which the computer sends out to the actuator. In order to seek the optimum control frequency for active control to surge, the different control effects to restrain the surge were investigated. Figure 9 stands for the different experimental results, and Figure 10 shows the dynamic behavior. It is shown that when the control frequency is less than $40 \mathrm{~Hz}$ the fluid oscillating amplitude decreases obviously. When the controlled frequency is in the range between 80 and $100 \mathrm{~Hz}$, it is impossible that the surge phenomenon is controlled actively.

From the results of the power spectrum analyses (Fig. 11), it is obvious that when the main controller frequency is less than $50 \mathrm{~Hz}$ the frequency value of the first harmonic wave is nearly equal to that of the actuator, this means that after the active control scheme on surge is carried out, the frequency component related to $3.26 \mathrm{~Hz}$ (for $B=0.34$ ) disappears, i.e., the surge phenomenon is restrained successfully. But there are other low frequency value in the system which is already not that of the surge. This frequency value is close to the frequency of the first harmonic wave of the actuator (compare Figs. 8(a) and 11). When the control frequency is larger than $50 \mathrm{~Hz}$, the low frequency value related to surge is stronger than the first harmonic wave of the actuator and its amplitude increases with the increase of the control frequency.

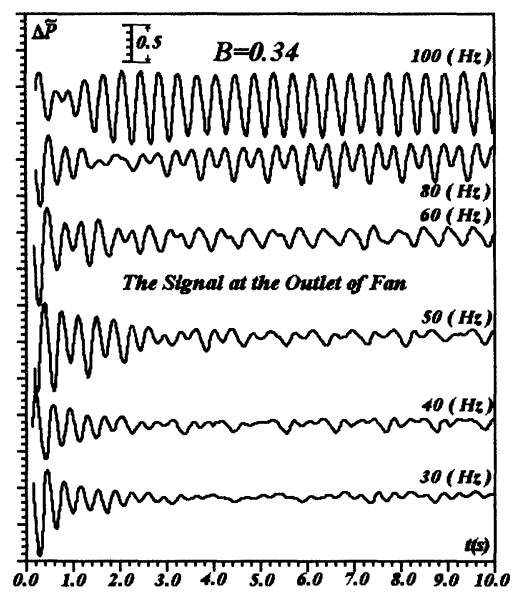

FIGURE 9 Controlled results of different frequencies at time domain. (The comparison between the different frequencies supplied by computer during the occurrence of surge.) 

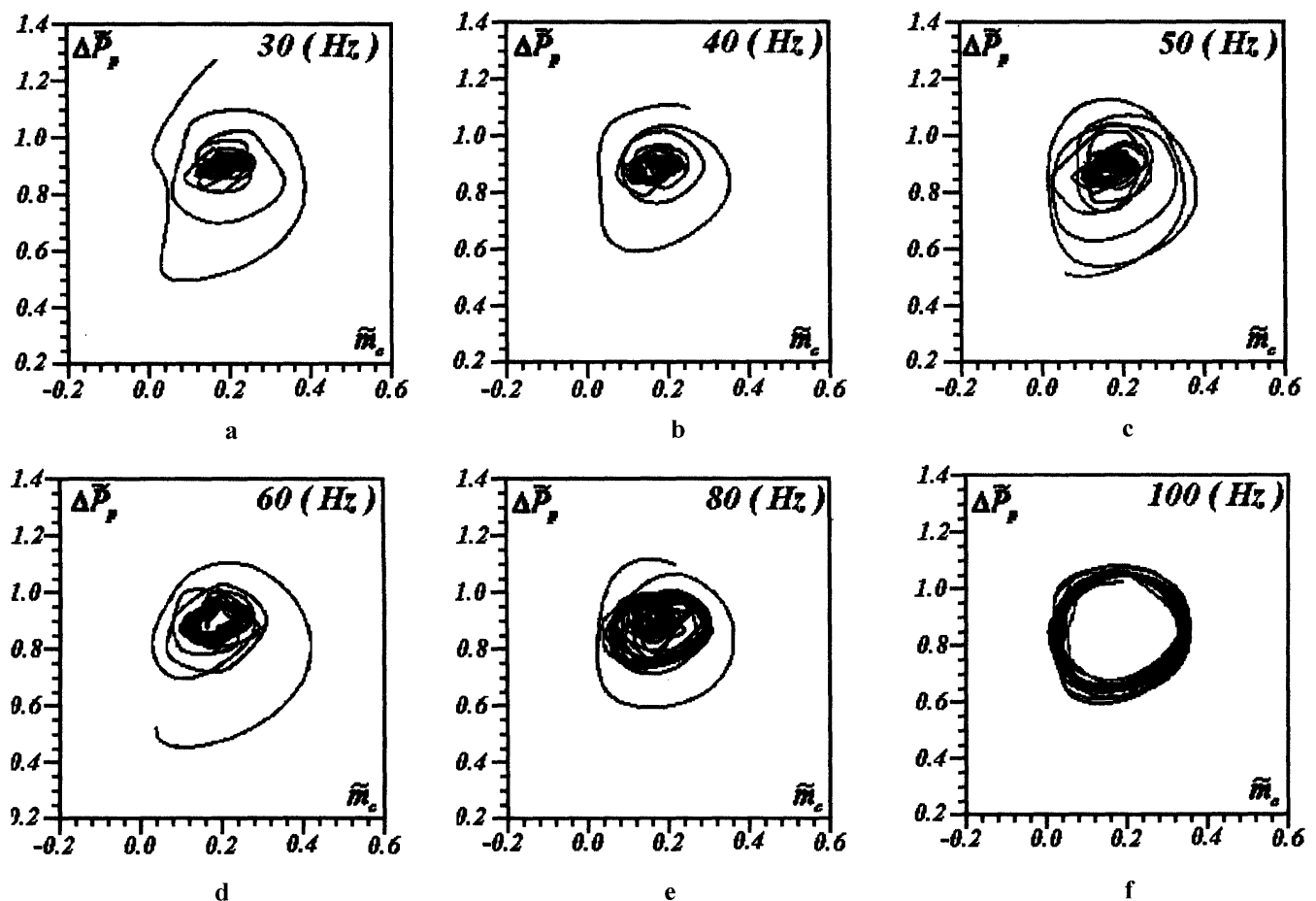

FIGURE 10 The dynamic characteristics of the different controller's frequency. (The group of figures shows the controlled effects in the view of both non-dimensional flow and pressure perturbation.)
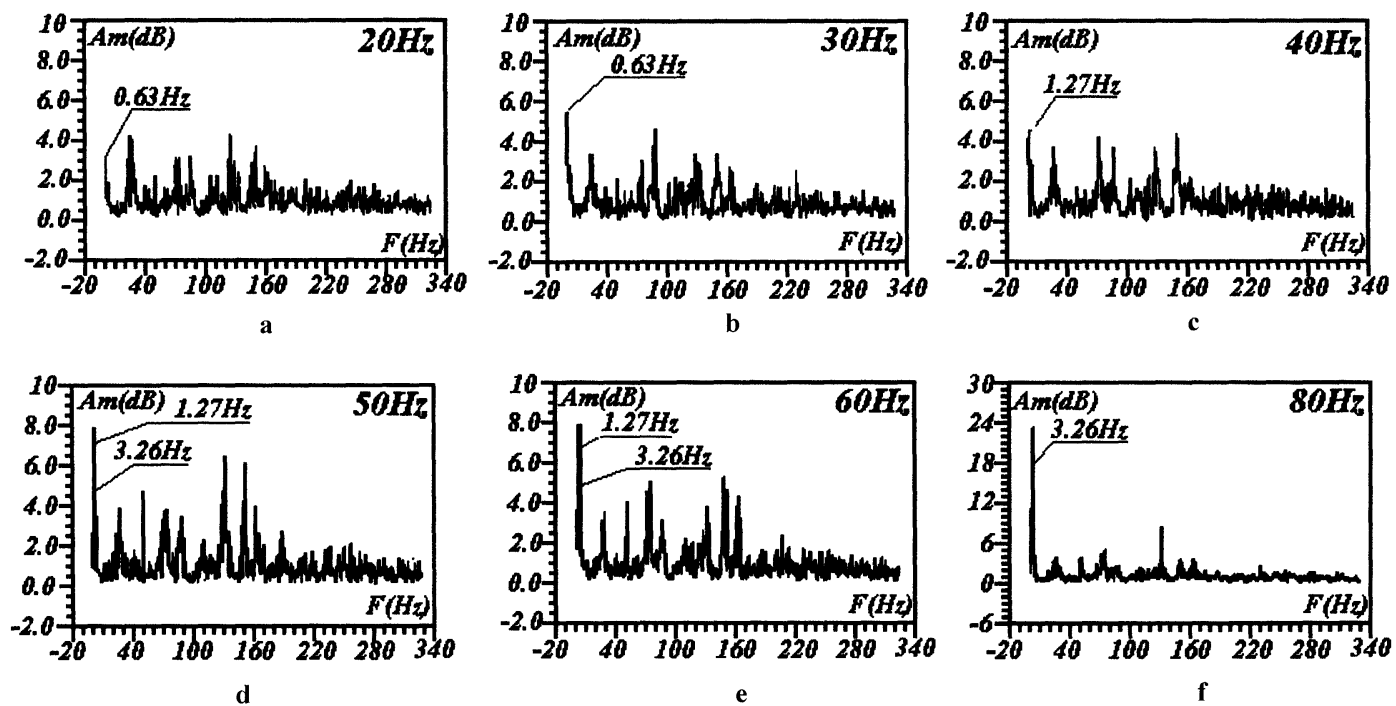

FIGURE 11 The power spectrum about the active control results to surge. (The group of figures demonstrates the coupled interaction between the main controller's frequency and the surge inherent frequency.) 
It is likely that this surge frequency is also enhanced by the higher harmonics of the actuator. On the whole, the optimum control frequency must be chosen between 20 and $40 \mathrm{~Hz}$. Therefore, after the active control scheme on surge is carried out, the frequency of the small fluid disturbance in the system is already not that of the surge. The frequency value is the frequency of the first harmonic wave of the actuator. The surge phenomenon is restrained successfully. While with the main frequency of the controller larger than $50 \mathrm{~Hz}$, the low frequency factors of the disturbances related to surge is stronger than the first harmonic wave of the actuator. The higher the main frequency value of controller is chosen, the larger the amplitude of the disturbance is caused. The optimum controlled frequency must be chosen between 20 and $40 \mathrm{~Hz}$.

\section{The Control Results of the Different Prescribed Criteria}

The prescribed criteria are set up in the range from 20 to $160 \mathrm{~dB}$. As soon as the amplitude of the first harmonic wave of the inception exceeds the preliminary criteria then does the actuator supply the small friendly disturbances. Figure 12 shows a group of the controlled results. When the prescribed criteria are set up at the lower values (from 20 to $50 \mathrm{~dB}$ ), the results of the active control are obvious, and the amplitude of the left perturbations is acceptable, when the prescribed criteria are set over $120 \mathrm{~dB}$, it takes long time to reach steadiness. For engineering applications, when a deep surge occurs in the system, the higher prescribed criteria results are not satisfying for implementing the active control plan. Therefore, the prescribed criterion should be set up to be less than $50 \mathrm{~dB}$. When the signal of the inception is identified, the plan of the active control is exerted immediately by operating the actuator.

\section{Entire Control Effects}

Figure 13 represents the results, it is shown that the amplitude of fluid oscillation is decreased

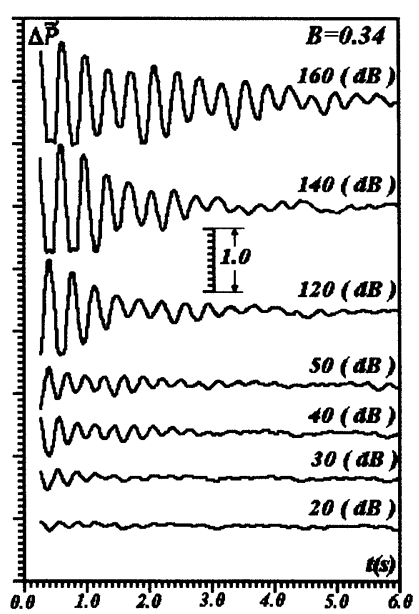

FIGURE 12 The controlled results in time domain. (The results of the active control to surge are contrasted with the prescribed amplitude criteria of pressure perturbation.)

dramatically after exerting the active control plan, at the same time, the oscillating loop of the surge draws back to the quasi-stable operating point rapidly.

\section{Extending Effects from Surge Boundary}

With the techniques of the active control, the operating ranges of the centrifugal fan. can be enlarged for passing through the surge boundary. In fact, the actual operating point draws back to the quasi-stable operating point after the surge is suppressed. Figure 14 shows the experimental results where the operating point passes through the surge boundary line. It is shown that the nondimensional flow quantity average value of the onset point of surge is about 0.196 . The limited nondimensional flow quantity average value of quasisteady point that is carried out with the technique of active control scheme is equal to 0.148 . Therefore, the steady operating range is expanded by approximately $24 \%$. If the area of the throttle is further decreased, the surge will be impelled rapidly (Fig. 15). It is shown that the actuator has no ability to control the new surge phenomena. The new unsteady point is considered to be new surge boundary point. 

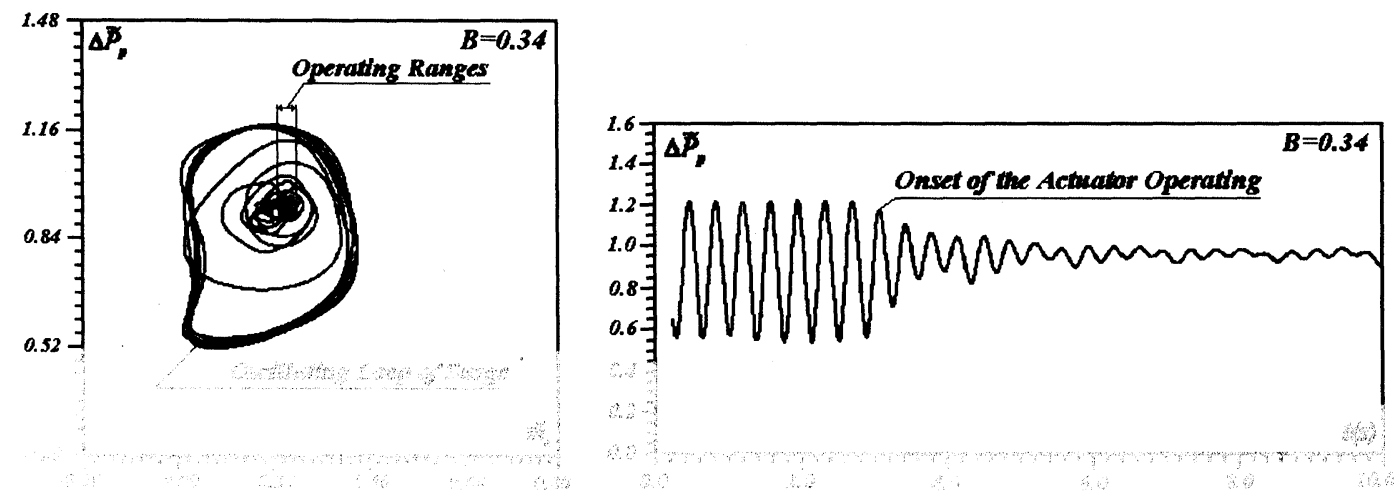

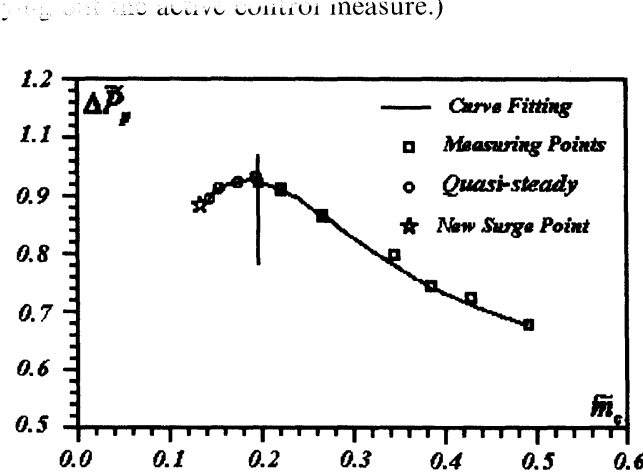

FIGURE 14 Extending surge boundary point. (The steady operating range is expanded by about $24 \%$ with the technique of active control.)

\section{CONCLUSIONS}

1. The dynamic behavior of surge in the actual centrifugal compression system is analyzed in detail. The experimental results show that the length of the time which the compression system enters surge compulsively is shorter than that of the time for spontaneous surge. The strength of the former is larger than that of the latter. The strength of the surge is positively proportional to the $B$ parameters, the frequency of surge is negatively proportional to the $B$ parameter.

2. The power spectrum analysis is applied to investigate the frequency behavior of the inception of surge. The experimental results reveal that the inception of surge can be diagnosed

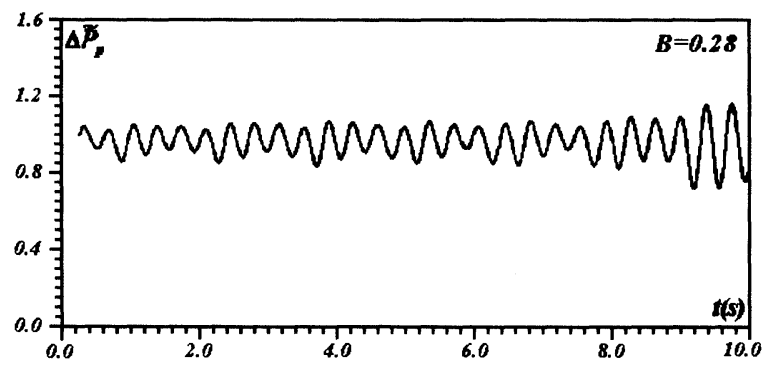

FIGURE 15 New surge point. (While the area of the throttle is further decreased the surge phenomena will be impelled rapidly.)

at both the outlet of the centrifugal fan and the plenum. With real-time analysis method, the inception of the surge is captured rapidly and the active control scheme is established.

3. The essential nonlinear characteristics with which the actuator draws the small friendly disturbances into the centrifugal compression system are investigated experimentally. These characteristics are important in designing the active control scheme and in understanding the control results.

4. The active control of surge is successfully realized in a range of control frequencies and prescribed amplitude criteria of the surge inception signal. The stable range where the actual centrifugal compression system operates is extended by $24 \%$ with the technique of the active control. 


\section{Acknowledgments}

This research project is funded by the Climb Project $B$ and the National Science Foundation of China. The two effective supports are greatly appreciated. The authors are also indebted to Mr. Qiao Xiaohui for his assistance on experiment and data acquisition and processing.

\section{NOMENCLATURE}

$\begin{array}{ll}B & \text { Greitzer parameter } \\ m & \text { Mass flow } \\ t(\mathrm{~s}) & \text { Time (second) } \\ \Delta P & \text { The pressure difference }\end{array}$

\section{Subscripts}

p Plenum

\section{Superscripts}

$\sim$ non-dimensional variable

\section{References}

Day, I.J. (1993a) Stall inception in axial flow compressors, ASME Journal of Turbomachinery, 115, 1-9.

Day, I.J. (1993b) Active suppression of rotating stall and surge in axial compressors, ASME Journal of Turbomachinery, 115 , 40-47.

Epstein, A.H., Ffowcs Williams, J.E. and Greitzer, E.M. (1989) Active suppression of aerodynamic instabilities in turbomachines, Journal of Propulsion and Power, 5, 204-211.

Ffowcs Williams, J.E. and Huang, X.Y. (1989) Active stabilization of compressor surge, Journal of Fluid Mechanics, 204, $245-262$.
Garnier, V.H., Epstein, A.H. and Greitzer, E.M. (1991) Rotating waves as a stall inception indication in axial compressors, ASME Journal of Turbomachinery, 113, 290-301.

Greitzer, E.M. (1976a) Surge and rotating stall in axial flow compressors, Part 1: Theoretical compression system model, ASME Journal of Engineering for Power, 98, 190-198.

Greitzer, E.M. (1976b) Surge and rotating stall in axial flow compressors, Part 2: Experimental results and comparison with theory, ASME Journal of Engineering for Power, 98 199-217.

Greitzer, E.M. (1981) The stability of dumping system - The 1980 Freeman scholar lecture, ASME Journal of Fluids Engineering, 103, 193-242.

Gysling, D.L., Dugundji, J., Greitzer, E.M. and Epstein, A.H. (1991) Dynamic control of centrifugal compressor surge using tailored structures, ASME Journal of Turbomachinery, 113, $710-722$.

Gysling, D.L. and Greitzer, E.M. (1995) Dynamic control of rotating stall in axial flow compressors using aeromechanical feedback, ASME Journal of Turbomachinery, 117, $307-319$.

Haynes, J.M., Hendricks, G.J. and Epstein, A.H. (1994) Active stabilization of rotating stall in a three-stage axial compressor, ASME Journal of Turbomachinery, 116, 226-239.

Hoying, D.A. (1993) Stall inception in a multistage high speed axial compressor, AIAA Paper, No. 93-2386.

Moore, F.K. and Greitzer, E.M. (1986a) A theory of post-stall transients in axial compression systems, Part 1: Development of equations, ASME Journal of Engineering for Gas Turbines and Power, 108, 68-76.

Moore, F.K. and Greitzer, E.M. (1986b) A theory of post-stall transients in axial compression systems, Part 2: Application, ASME Journal of Engineering for Gas Turbines and Power, 108, 231-239.

Paduano, J.P., Epstein, A.H., Valavani, L., Longely, J.P., Greitzer, E.M. and Guenette, G.R. (1993) Active control of rotating stall in a low speed compressor, ASME Journal of Turbomachinery, 115, 48-56.

Pinsley, J.E., Guenette, G.R., Epstein, A.H. and Greitzer, E.M. (1991) Active stabilization of centrifugal compressor surge, ASME Journal of Turbomachinery, 113, 723-732.

Simon, J.S., Valavani, L., Epstein, A.H. and Greitzer, E.M. (1993) Evaluations of approaches to active compressor surge stabilization, ASME Journal of Turbomachinery, 115, $57-67$.

Tryfonidis, H., Etchevers, O., Paduano, J.D., Epstein, A.H and Hendricks, G.J. (1995) Pre-stall behavior of several high-speed compressors, ASME Journal of Turbomachinery, 117, 62-80 


\section{ait \\ ENERGY MATERIALS}

M A N E Y publishing

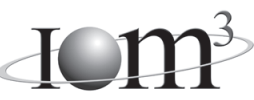

\section{Materials Science \& Engineering for Energy Systems}

Maney Publishing on behalf of the Institute of Materials, Minerals and Mining

The Institute of Materials, Minerals \& Mining

Economic and environmental factors are creating ever greater pressures for the efficient generation, transmission and use of energy. Materials developments are crucial to progress in all these areas: to innovation in design; to extending lifetime and maintenance intervals; and to successful operation in more demanding environments. Drawing together the broad community with interests in these areas, Energy Materials addresses materials needs in future energy generation, transmission, utilisation, conservation and storage. The journal covers thermal generation and gas turbines; renewable power (wind, wave, tidal, hydro, solar and geothermal); fuel cells (low and high temperature); materials issues relevant to biomass and biotechnology; nuclear power generation (fission and fusion); hydrogen generation and storage in the context of the 'hydrogen economy'; and the transmission and storage of the energy produced.

As well as publishing high-quality peer-reviewed research, Energy Materials promotes discussion of issues common to all sectors, through commissioned reviews and commentaries. The journal includes coverage of energy economics and policy, and broader social issues, since the political and legislative context influence research and investment decisions.

\section{CALL FOR PAPERS}

Contributions to the journal should be submitted online at http://ema.edmgr.com

To view the Notes for Contributors please visit: www.maney.co.uk/journals/notes/ema

Upon publication in 2006, this journal will be available via the Ingenta Connect journals service. To view free sample content online visit: www.ingentaconnect.com/content/maney

For further information please contact:

Maney Publishing UK

Tel: +44 (0)113 2497481 Fax: +44 (0)1132486983 Email: subscriptions@maney.co.uk

or

Maney Publishing North America

Tel (toll free): 8662975154 Fax: 6173546875 Email: maney@maneyusa.com

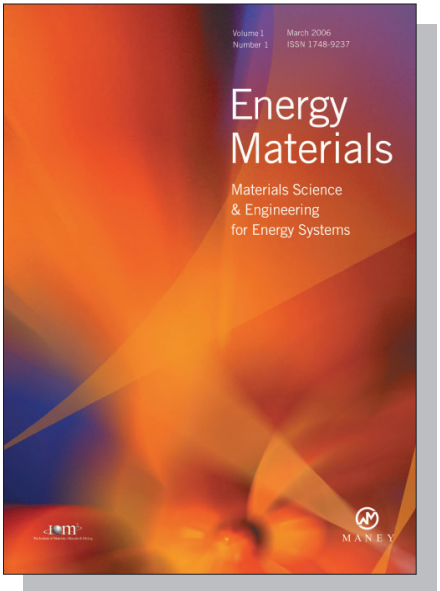

EDITORS

Dr Fujio Abe

NIMS, Japan

Dr John Hald, IPL-MPT, Technical University of Denmark, Denmark

Dr R Viswanathan, EPRI, USA

\section{SUBSCRIPTION INFORMATION}

Volume 1 (2006), 4 issues per year

Print ISSN: 1748-9237 Online ISSN: 1748-9245

Individual rate: $£ 76.00 / U S \$ 141.00$

Institutional rate: $£ 235.00 /$ US $\$ 435.00$

Online-only institutional rate: $£ 199.00 / U S \$ 367.00$

For special $\mathrm{IOM}^{3}$ member rates please email

subscriptions@maney.co.uk

\section{For further information or to subscribe online please visit www.maney.co.uk}



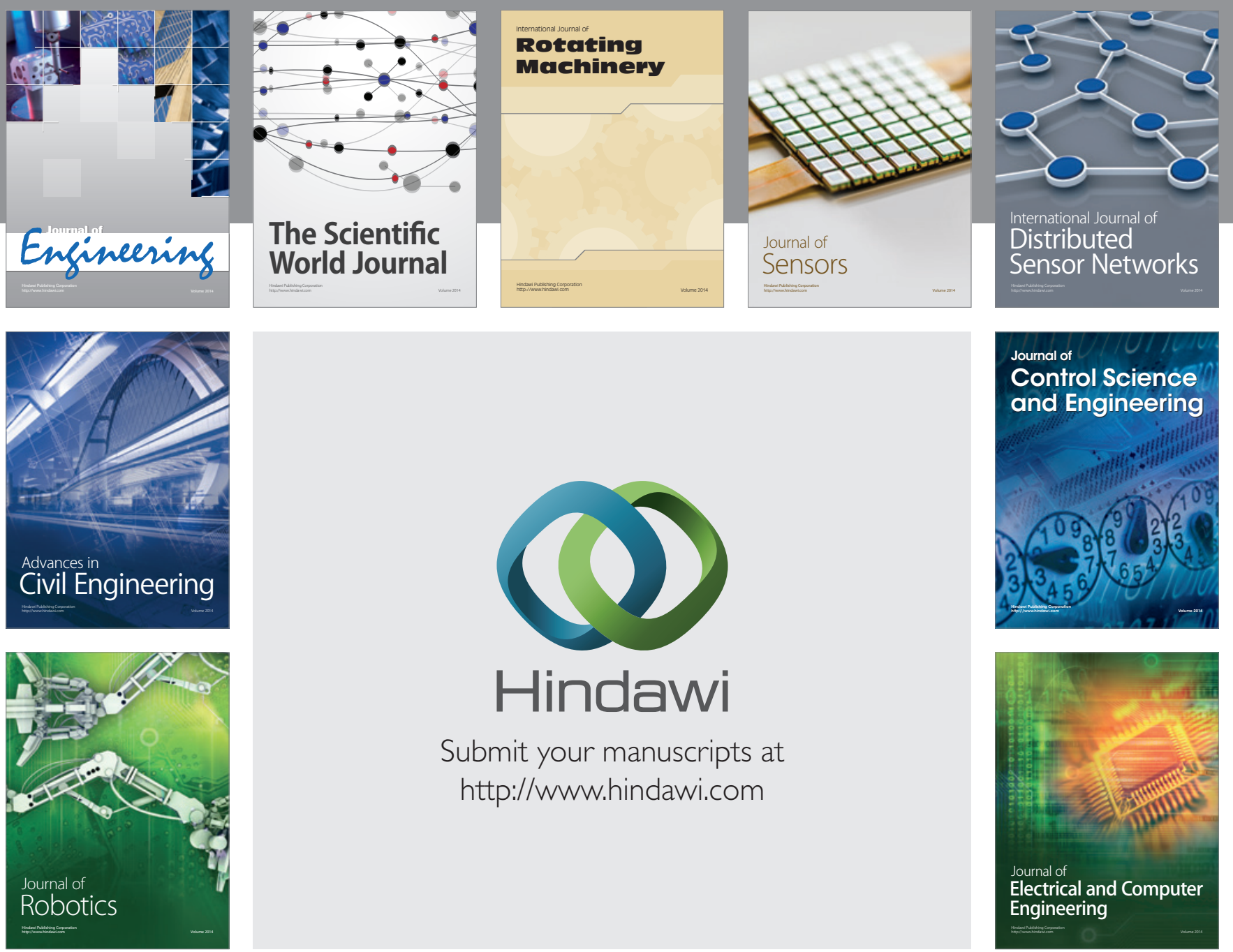

Submit your manuscripts at

http://www.hindawi.com
